\title{
Priority and Proportionality in Bankruptcy
}

Flores-Szwagrzak, Karol; García-Segarra, Jaume; Ginés-Vilar, Miguel

Document Version

Accepted author manuscript

Published in:

Social Choice and Welfare

DOI:

10.1007/s00355-019-01219-0

Publication date:

2020

License

Unspecified

Citation for published version (APA):

Flores-Szwagrzak, K., García-Segarra, J., \& Ginés-Vilar, M. (2020). Priority and Proportionality in Bankruptcy.

Social Choice and Welfare, 54(4), 559-579. https://doi.org/10.1007/s00355-019-01219-0

Link to publication in CBS Research Portal

\section{General rights}

Copyright and moral rights for the publications made accessible in the public portal are retained by the authors and/or other copyright owners and it is a condition of accessing publications that users recognise and abide by the legal requirements associated with these rights.

Take down policy

If you believe that this document breaches copyright please contact us (research.lib@cbs.dk) providing details, and we will remove access to the work immediately and investigate your claim. 


\section{Priority and Proportionality in Bankruptcy Karol Flores-Szwagrzak, Jaume García-Segarra, and Miguel Ginés-Vilar}

Journal article (Accepted version*)

\section{Please cite this article as:}

Flores-Szwagrzak, K., García-Segarra, J., \& Ginés-Vilar, M. (2020). Priority and Proportionality in Bankruptcy. Social Choice and Welfare, 54(4), 559-579. https://doi.org/10.1007/s00355-019-01219-0

This is a post-peer-review, pre-copyedit version of an article published in Social Choice and Welfare. The final authenticated version is available online at:

DOl: https://doi.org/10.1007/s00355-019-01219-0

* This version of the article has been accepted for publication and undergone full peer review but has not been through the copyediting, typesetting, pagination and proofreading process, which may lead to differences between this version and the publisher's final version AKA Version of Record.

Uploaded to CBS Research Portal: May २०२० 


\title{
Priority and proportionality in bankruptcy*
}

\author{
Karol Flores-Szwagrzak $\left(^{*}\right)^{\dagger} \quad$ Jaume García-Segarra ${ }^{\ddagger} \quad$ Miguel Ginés-Vilar ${ }^{\S}$
}

September 10, 2019

\begin{abstract}
We study the problem of distributing the liquidation value of a bankrupt firm among its creditors (O'Neill, 1982; Aumann and Maschler, 1985). Reallife distribution rules prioritize predetermined creditor groups, dividing the amount assigned to each group proportionally to claims. We provide the first axiomatic characterization of such rules. In addition to the classical consistency and continuity axioms, these rules are characterized by the following properties: (i) bankruptcy problems with the same claims and where each claimant's award is positive in each problem can be solved either jointly or separately without altering the recommended awards, (ii) a dual property specifying that bankruptcy problems with the same claims and where each claimant's loss is positive can be solved either jointly or separately without altering the recommended awards.
\end{abstract}

Keywords: Bankruptcy problem, Proportional rule, Priority JEL classification: D70, D63, D71

\section{Introduction}

When a firm goes bankrupt, how should its liquidation value be divided among its creditors? In bankruptcy proceedings, creditors are prioritized according to their

*We are grateful to the associate editor and a referee for a number of comments and requests that have significantly improved the material herein. We also thank Carlos Alós-Ferrer and William Thomson for extensive comments. Karol Flores-Szwagrzak gratefully acknowledges financial support from the Independent Research Fund Denmark (Grant ID: DFF-6109-00132).

${ }^{\dagger}$ Copenhagen Business School. E-mail: ksz.eco@cbs.dk

${ }^{\ddagger}$ University of Cologne. E-mail: jaume.garcia-segarra@uni-koeln.de

$\S$ University Jaume I of Castellón. E-mail: mgines@uji.es 
individual characteristics: for example, employees may be first in line, tax authorities second, and other creditors third. Within each creditor class, the available amount is distributed proportionally to the creditors' claims.

Bankruptcy settlement follows this general structure in many countries. Naturally, countries differ in how they prioritize different creditors. However, the assets available to creditors who are deemed to have equal priority are adjudicated proportionally to their claims. For example, the United States Bankruptcy Code specifies how different creditors are sorted into priority classes ( $\$ 507$ in the Title 11 of the United States Code) and that "the payments within each priority class shall be made pro rata among claims of the same class" ( $\S 726 \mathrm{~b})$. European bankruptcy law also specifies proportionality among creditors of the same standing where the standing refers to the position in the priority order (Council Regulation EC No 1346/2000 of May 29th on insolvency proceedings).

Though bankruptcy rules combining priority and proportionality are common, the axiomatic literature on bankruptcy rules lacks a characterization of these "priorityaugmented proportional rules." ${ }^{1}$ The challenge is that these rules combine two seemingly contradictory normative principles with very different formal content: Priority stresses the importance of the creditors' individual characteristics, beyond their claims. These characteristics are formally outside of the model. ${ }^{2}$ Proportionality, in contrast, stresses that only the size of the claims matters and not any other characteristics of their holders; every dollar claimed should be treated equally (Young, 1994).

We address this challenge by identifying four formal properties of the proportional rule compatible with priority structures that characterize these priority-augmented proportional rules. Two of these properties, "continuity" and "consistency," are classical and satisfied by all major families of rules (those of Young, 1987; Moulin, 2000; Stovall, 2014a,b). Continuity requires that small changes in the claims or endowment do not result in significant changes in the recommended distribution; consistency requires that, if a distribution is considered desirable for a group of creditors, it remains so when restricted to each creditor subgroup.

The other two properties, "restricted additivity" and "dual restricted additivity," are new. Restricted additivity concerns creditors' incentives in specifying the assets

\footnotetext{
${ }^{1}$ For surveys, see Moulin (2002) and Thomson (2003, 2015); Thomson (2019) provides an in depth treatment. The proportional rule, without priority structures, has been extensively studied; characterizations include those of O'Neill (1982), Chun (1988), Young (1988), de Frutos (1999), Moreno-Ternero (2006), Ju et al. (2007), Csoka and Herings (2016), and Thomson (2016).

${ }^{2}$ See Moulin (2000) and Stovall (2014a) for detailed motivation of the importance of acknowledging individual claimant characteristics in the classical bankruptcy problem.
} 
and claims to be adjudicated in a bankruptcy court. It eliminates incentives for litigation to consolidate assets and claims from multiple cases. Since it may not be feasible to consolidate claims across arbitrary bankruptcy cases, we do not impose the condition on arbitrary cases. We only impose it on bankruptcy problems where (i) the standing of creditors is the same and (ii) all creditors receive positive awards, none is marginalized in the adjudication. Why (ii)? Estate division offers a transparent motivating example: A mother and a father pass away within a short period of time leaving behind their estates to their children. The forgiving mother leaves a will specifying her money goes to all of her children; the resentful father excludes a rebellious child from inheriting anything. In respecting the wishes of the deceased, the two estates and the claims on these should not simply be merged and settled jointly. On the other hand, if the father's will is like the mother's, the two estate problems may as well be consolidated and settled as one.

To better explain dual restricted additivity, first recall the central "duality" principle for bankruptcy problems (Aumann and Maschler, 1985). It captures the idea that instead of distributing what is available and recommending awards we can resolve a bankruptcy case by distributing the shortfall - the sum of the claims minus the available amount - and recommend losses relative to claims across creditors. Dual restricted additivity is then the requirement that the consolidation of two bankruptcy cases can only go forward if (i) the standing of creditors is the same in both cases and (ii) all creditors endure losses, none is marginalized in the dual adjudication. ${ }^{3}$

Even though the priority-augmented proportional rules have not been characterized before, they belong to the class of asymmetric rationing rules (Moulin, 2000). These rules also involve different priority groups. The amount available to each priority group is distributed using the proportional rule, a weighted version of the constrained equal awards rule, or a weighted version of the constrained equal losses rule. The sub-class of asymmetric rationing rules using either a weighted constrained equal awards rule or a constrained equal losses rule within each priority group has been characterized using standard axioms (Flores-Szwagrzak, 2015). In this paper, we characterize the rules using the proportional rule within each priority group.

The remainder of this paper is organized as follows: Section 2 introduces the model and the priority-augmented proportional rules. Section 3 contains the axiomatic analysis and our results. We also provide three additional characterizations of the PAP rules. Section 4 concludes. An Appendix contains the proofs.

\footnotetext{
${ }^{3}$ The rationale for (ii) is dual to that for restricted additivity.
} 


\section{Definitions}

\section{$2.1 \quad$ Model}

An endowment of a divisible resource is to be distributed among a finite group of claimants. These groups are drawn from a countable set of "potential claimants" that we identify with $\mathbb{N}$, the natural numbers. ${ }^{4}$ Let $\mathcal{N}$ denote the collection of finite subsets of $\mathbb{N}$. For each $N \in \mathcal{N}$, a claims problem is a pair consisting of a profile of claims and an endowment $(c, E) \in \mathbb{R}_{+}^{N} \times \mathbb{R}_{+}$such that $\sum_{N} c_{i} \geq E$. For each $N \in \mathcal{N}$, let $\mathcal{C}^{N}$ denote the class of claims problems involving the claimants in $N$. An allocation for the problem $(c, E) \in \mathcal{C}^{N}$ is a profile $z \in \mathbb{R}^{N}$ such that $\sum_{N} z_{i}=E$ and, for each $i \in N, 0 \leq z_{i} \leq c_{i}$; we refer to $z_{i}$ as the award of claimant $i$. Let $Z(c, E)$ denote the collection of all allocations for claims problem $(c, E)$. A rule is a function $f$ recommending an allocation for each possible claims problem: for each $N \in \mathcal{N}$ and each $(c, E) \in \mathcal{C}^{N}, f(c, E) \in Z(c, E) .^{5}$

\subsection{Rules}

We start by introducing three classical rules. The proportional rule, $P$, awards each claimant an equal fraction of her claim. Formally, for each $N \in \mathcal{N}$, each $(c, E) \in \mathcal{C}^{N}$, and each $i \in N$,

$$
P_{i}(c, E)=\lambda c_{i} \quad \text { where } \quad \lambda=\frac{E}{\sum_{N} c_{j}}
$$

whenever the sum of the claims is positive, otherwise each claimant receives a null award. The constrained equal awards rule, $C E A$, is such that for each $N \in \mathcal{N}$, each $(c, E) \in \mathcal{C}^{N}$, and each $i \in N, C E A_{i}(c, E)=\min \left\{c_{i}, \lambda\right\}$ where $\lambda$ is chosen so that $\sum_{N} \min \left\{c_{i}, \lambda\right\}=E$. The constrained equal losses rule, $C E L$, is such that for each $N \in \mathcal{N}$, each $(c, E) \in \mathcal{C}^{N}$, and each $i \in N, C E L_{i}(c, E)=\max \left\{0, c_{i}-\lambda\right\}$ where $\lambda$ is chosen so that $\sum_{N} \max \left\{0, c_{i}-\lambda\right\}=E$.

When a rule varies continuously with the endowment, as is the case with the above rules, its recommended awards form a continuous path in the space of awards. In this case, a useful way of describing a rule is through this path. Formally, the path of awards of a rule $f$ is, for each $N \in \mathcal{N}$ and each $c \in \mathbb{R}_{+}^{N}$, the locus

\footnotetext{
${ }^{4}$ Mathematical notation is as follows: $\left\{Y_{i}\right\}_{i \in I}$ denotes a family of sets $Y_{i}$ indexed by $I$ and $Y^{I}=\mathrm{X}_{i \in I} Y_{i}$. For each $y \in Y^{I}$ and each $J \subseteq I, y_{J}$ denotes the projection of $y$ onto $Y^{J}$. For each pair $x, y \in \mathbb{R}^{I}, x \geq y$ means that $x_{i} \geq y_{i}$ for each $i \in I$ and $x>y$ means that $x_{i}>y_{i}$ for each $i \in I$.

${ }^{5}$ As usual, for each $N \in \mathcal{N}$, each $c \in \mathbb{R}_{+}^{N}$, and each $i \in N$, we will use $c_{-i}$ to denote the more cumbersome $c_{N \backslash\{i\}}$.
} 


$$
\left\{x \in \mathbb{R}_{+}^{N}: \exists E \in\left[0, \sum_{N} c_{i}\right], x=f(c, E)\right\} .
$$

We now introduce the notion of a priority relation over the potential claimants. Formally, a priority relation is a complete and transitive binary relation $\succsim$ on $\mathbb{N}$; its asymmetric part is denoted by $\succ$ while its symmetric part is denoted by $\sim$. The statement $i \succ j$ is read as " $i$ has priority over $j$." Since each $N \in \mathcal{N}$ is finite, $\succsim$ induces a partition of $N$ into finitely many priority classes as follows:

$$
\begin{aligned}
& N_{1}=\{i \in N: \text { for each } j \in N, i \succsim j\} \\
& N_{2}=\left\{i \in N \backslash N_{1}: \text { for each } j \in N \backslash N_{1}, i \succsim j\right\} \\
& N_{3}=\left\{i \in N \backslash\left[N_{1} \cup N_{2}\right]: \text { for each } j \in N \backslash\left[N_{1} \cup N_{2}\right], i \succsim j\right\}
\end{aligned}
$$

For each $N \in \mathcal{N}$, we will denote the partition of $N$ into $k \geq 1$ priority classes by $N_{1}, \ldots, N_{k}$, suppressing the dependence on $\succsim$ whenever there is no room for confusion.

For each priority relation $\succsim$, the priority-augmented proportional rule associated to $\succsim$, is the rule denoted by $P^{\succsim}$ such that for each $N \in \mathcal{N}$ and each $(c, E) \in \mathcal{C}^{N}$,

for each $i \in N_{1}, \quad P_{i}^{\succsim}(c, E)=P_{i}\left(c_{N_{1}}, E_{1}\right) \quad$ where $\quad E_{1}=\min \left\{E, \sum_{N_{1}} c_{j}\right\}$;

for each $i \in N_{2}, \quad P_{i}^{\succsim}(c, E)=P_{i}\left(c_{N_{2}}, E_{2}\right) \quad$ where $\quad E_{2}=\min \left\{E-E_{1}, \sum_{N_{2}} c_{j}\right\}$;

for each $i \in N_{3}, \quad P_{i}^{\succsim}(c, E)=P_{i}\left(c_{N_{3}}, E_{3}\right) \quad$ where $\quad E_{3}=\min \left\{E-E_{1}-E_{2}, \sum_{N_{3}} c_{j}\right\}$;

We will refer to the rules defined in this way, for each possible priority relation, as priority-augmented proportional rules or, for brevity, PAP rules. We conclude this section introducing a rule to illustrate the logical independence of the axioms in the characterizations of the PAP rules. The larger-claims-first rule, $L C F$, lexicographically favors claimants holding larger claims: The claimants with the largest claim each receive their claim if possible or divide the endowment equally among themselves; if a part of the endowment is left over, the claimants with the second largest claim receive their claim if possible or divide the remaining endowment equally; the distribution continues in this way until the endowment is exhausted. 


\section{Axiomatic analysis}

We start with a central principle in the theory of distributive justice, consistency. It has been a key axiom in the analysis of claims problems dating back to the seminal contributions of Aumann and Maschler (1985) and Young (1987, 1988). It captures that idea that if an allocation is viewed as desirable, then its restriction to each subgroup of claimants ought to be considered desirable as well. This is expressed as follows: Given a rule, a claims problem, and its recommended allocation for the problem, consider the departure of some claimants with their awards; the requirement is that, upon reassessing the distribution of the available amount, the rule recommends, for each remaining claimant, the same amount it did to begin with.

Consistency: For each $N \in \mathcal{N}$, each $J \subseteq N$, and each $(c, E) \in \mathcal{C}^{N}, f_{J}(c, E)=$ $f\left(c_{J}, \sum_{J} x_{j}\right)$.

A weaker condition, bilateral consistency, restricts the conclusion to twoclaimant groups (when the cardinality of $J$ is two in the definition of consistency).

Another classical requirement is for a rule's recommendations not to change significantly in response to small changes in the claims and endowment. Thus, the rule ought to vary continuously on the claims problem with respect to the Euclidean topology (Young, 1987).

Continuity: For each sequence $\left\{\left(c^{n}, E^{n}\right)\right\}$ of elements in $\mathcal{C}^{N}$ and each $(c, E) \in \mathcal{C}^{N}$, if $\left(c^{n}, E^{n}\right) \rightarrow(c, E)$, then $f\left(c^{n}, E^{n}\right) \rightarrow f(c, E){ }^{6}$

Consistency and continuity are standard axioms. They are satisfied by the constrained equal awards rule, the constrained equal losses, the Talmud, and the proportional rules as well as the parametric rules (Young, 1987), the equal sacrifice rules (Young, 1988), the asymmetric rationing rules (Moulin, 2000), the asymmetric parametric rules (Stovall, 2014a), and the monotone path rules (Stovall, 2014b).

We now introduce a new additivity axiom. Additivity conditions have been studied widely in surplus and cost sharing (Moulin, 2002) and in cooperative game theory, particularly in the characterization of the Shapley value (Shapley, 1953; Roth, 1988). For claims problems, the most straightforward additivity requirement is that given two claims problems involving a group of claimants, a rule can, equivalently, recommend allocations for each problem separately or do so jointly, by first adding the

\footnotetext{
${ }^{6}$ Two weaker continuity properties can be formulated. Endowment continuity specifies that a rule varies continuously on the endowment but not necessarily on claims. Formally, for each sequence $\left\{\left(c^{n}, E^{n}\right)\right\}$ of elements in $\mathcal{C}^{N}$ and each $(c, E) \in \mathcal{C}^{N}$, if $c^{n} \rightarrow c$ and $E^{n}=E$, then $f\left(c^{n}, E^{n}\right) \rightarrow f(c, E)$. Claims continuity specifies that a rule varies continuously on claims but not necessarily on the endowment. Formally, for each sequence $\left\{\left(c^{n}, E^{n}\right)\right\}$ of elements in $\mathcal{C}^{N}$ and each $(c, E) \in \mathcal{C}^{N}$, if $c^{n}=c$ and $E^{n} \rightarrow E$, then $f\left(c^{n}, E^{n}\right) \rightarrow f(c, E)$.
} 
claims of agents across the two problems as well as the endowments. Though it is a natural requirement, there is no rule satisfying it (Bergantiños and Vidal-Puga, 2004). However, weaker and meaningful additivity properties that are satisfied by a number of rules have been studied (Moulin, 1987; Chun, 1988; Bergantiños and Méndez-Naya, 2001; Alcalde et al., 2014; Harless, 2017; Arin et al., 2017); these will be discussed in more detail below.

To formulate our additivity axiom, we first observe that claims problems with the same claimants and claims can either be solved separately or jointly. Additivity can then be satisfied. Imposing additivity in these cases expresses the idea that, when the standing of claimants in two problems is the same, consolidating the problems is more natural than when claims differ arbitrarily, rendering the problems qualitatively distinct. Moreover, we insist on additivity only in situations where all claimants receive positive awards and are thus not ignored by the adjudication procedure. This elicits the allocation behavior of a rule when it is effectively balancing the welfare of all claimants.

Restricted additivity: For each $c \in \mathbb{R}_{+}^{N}$ and each pair $E, E^{\prime} \in\left[0, \sum_{N} c_{i}\right]$ such that $f(c, E)>0$ and $f\left(c, E^{\prime}\right)>0, f(c, E)+f\left(c, E^{\prime}\right)=f\left(c+c, E+E^{\prime}\right)$.

Restricted additivity is satisfied by several well-known rules beyond the PAP rules. The CEL rule, the priority-augmented CEL rules, and even the priorityaugmented weighted CEL rules characterized by (Flores-Szwagrzak, 2015) satisfy this property.

To introduce our next axiom, we first discuss the notion of "duality" (Aumann and Maschler, 1985). It is one of the most useful concepts describing the structure of the space of rules (Thomson, 2015) and particularly fruitful in our setting; it describes the connection between the allocation of awards and losses in a claims problem: a claimant's award determines her loss, and conversely. Indeed, we can define a rule distributing the endowment $(E)$ in terms of its "dual" rule distributing the shortfall $\left(\sum_{N} c_{i}-E\right)$. Formally, the dual of $f, f^{d}$, is defined by,

$$
\text { for each }(c, E) \in \mathcal{C}^{N}, \quad f^{d}(c, E)=c-f\left(c, \sum_{N} c_{i}-E\right) \text {. }
$$

Remark 1. The dual of a PAP rule associated to $\succsim$ is the PAP rule associated to the "reverse" priority relation. ${ }^{7}$

Two axioms are dual if whenever a rule satisfies one of them, its dual satisfies the other one (Thomson, 2003, 2015). Restricted additivity enables claims problems to be aggregated conditional on every claimant receiving positive awards in the original

\footnotetext{
${ }^{7}$ For each pair $i, j \in \mathbb{N}$, let $i \succsim^{r} j$ whenever $j \succsim i$. Then, the dual of $P \succsim$ is $P^{\succsim^{r}}$.
} 
problems; the dual axiom enables aggregation conditional on every claimant making positive losses in the original problems. The axiom dual to restricted additivity can thus be stated as follows:

Dual restricted additivity: For each $c \in \mathbb{R}_{+}^{N}$ and each pair $E, E^{\prime} \in\left[0, \sum_{N} c_{i}\right]$ such that $f(c, E)<c$ and $f\left(c, E^{\prime}\right)<c, f(c, E)+f\left(c, E^{\prime}\right)=f\left(c+c, E+E^{\prime}\right)$.

Like restricted additivity, the dual axiom specifies that additivity applies when the claims in the to be consolidated problems are the same. Moreover, we insist on additivity only when all claimants receive positive losses and are thus not ignored by the loss adjudication procedure. This captures the effective behavior of a rule when it affects the losses of all claimants.

Dual restricted additivity is satisfied by several well-known rules beyond the PAP rules. The CEA rule, the priority-augmented CEA rules, and even the priorityaugmented weighted CEA rules characterized by Flores-Szwagrzak (2015) satisfy this property.

Our main result is that the PAP rules are characterized by the above axioms:

Theorem 1. The PAP rules are the only bilaterally consistent and continuous rules satisfying restricted additivity and dual restricted additivity.

\subsection{Proof overview}

The first step shows that a continuous rule satisfying restricted additivity and its dual satisfies weaker versions of "endowment linearity" (Chun, 1988), the requirement on a rule that the awards vector should be a linear function of the endowment.

Endowment linearity: For each $c \in \mathbb{R}_{+}^{N}$, each pair $E, E^{\prime} \in\left[0, \sum_{N} c_{i}\right]$, and each $\lambda \in[0,1], f\left(c, \lambda E^{\prime}+(1-\lambda) E\right)=\lambda f\left(c, E^{\prime}\right)+(1-\lambda) f(c, E)$.

Endowment linearity single-handedly characterizes the proportional rule (Chun, 1988). In contrast, a continuous rule satisfying restricted additivity is a linear function of the endowment provided that the endowment varies in a range where all claimants' awards are positive; similarly, a continuous rule satisfying dual restricted additivity is a linear function of the endowment provided that the endowment varies in a range where all claimants' losses are positive (Lemma 3 in the Appendix).

The second step shows that only two continuous two-claimant rules satisfy our weaker endowment linearity properties: the proportional rule and the rule fully prioritizing a predetermined claimant (Lemma 4 in the Appendix).

The last step shows that the only bilaterally consistent rule compatible with these two-claimant allocations is a PAP rule. Indeed, if $f$ denotes a bilaterally consistent 
rule that coincides, in two-claimant problems, with either the proportional rule or a rule fully prioritizing one of the claimants, we can define a priority relation $\succsim$ on $\mathbb{N}$ : For each pair $i, j \in \mathbb{N}$, let $i \sim j$ if the rule used to settle their claims problem is the proportional rule and let $i \succ j$ if the rule fully prioritizes $i$. By bilateral consistency, $\succsim$ is transitive. ${ }^{8}$ The proof of $f=P \succsim$ uses an "Elevator Lemma" argument (Thomson, 2011) enabled by the fact that $P \succsim$ satisfies a property known as "converse consistency" (see Appendix A.1) specifying that, if an allocation is such that its restriction is chosen by the rule for each two-claimant subgroup, then the allocation is chosen by the rule for the whole group.

\subsection{Characterizations of the proportional rule}

Imposing additional axioms in Theorem 1 characterizes the proportional rule. The first of these axioms requires that the allocation of the endowment and the shortfall should be treated symmetrically. As formalized by Aumann and Maschler (1985), the dual of a rule be the rule itself:

Self-duality: For each $(c, E) \in \mathcal{C}^{N}, f(c, E)=c-f\left(c, \sum_{N} c_{i}-E\right)$.

Many rules are self-dual, including the proportional, Talmud, and random arrival rules.

The central equity criterion for claims problems is that claimants with equal claims ought to receive equal awards.

Equal treatment of equal claims: For each $(c, E) \in \mathcal{C}^{N}$ and each pair $i, j \in N$, $c_{i}=c_{j}$ implies that $f_{i}(c, E)=f_{j}(c, E)$.

We can also specify that, for each claimant, there is a claim she can hold that will ensure her a positive award. For the condition to be more meaningful, we will also require that the endowment falls within a range that ensures that the award of the claimant is not positive simply due to the feasibility constraints:

Minimal compensation: For each $N \in \mathcal{N}$, each $i \in N$, each $(c, E) \in \mathcal{C}^{N}$ such that $E \in\left(0, \sum_{N \backslash\{i\}} c_{j}\right]$, there is $c_{i}^{\prime}>0$ such that $f_{i}\left(c_{i}^{\prime}, c_{-i}, E\right)>0$.

In fact, the only PAP rule that satisfies any one of the above axioms is the proportional rule.

Corollary 1. The proportional rule is the only rule satisfying the four axioms in Theorem 1 and any one of the following axioms: self-duality, equal treatment of equal claims, or minimal compensation.

\footnotetext{
${ }^{8}$ See the Proof of Theorem 1 for the argument.
} 


\subsection{Alternative characterizations of the PAP rules}

Theorem 2 below provides three further characterizations of the PAP rules. These replace one or both of our additivity axioms by weak forms of known axioms only satisfied by the proportional rule, "endowment additivity" (Moulin, 1987; Chun, 1988) and "multiplicative invariance" (Marchant, 2008).

Endowment additivity requires that a claims problem can be solved either directly or by splitting the endowment into two claims problems, each maintaining the same claims as in the original problem.

Endowment additivity: For each $(c, E) \in \mathcal{C}^{N}$ and each pair $E^{\prime}, E^{\prime \prime} \in \mathbb{R}_{+}$such that $E=E^{\prime}+E^{\prime \prime}, f(c, E)=f\left(c, E^{\prime}\right)+f\left(c, E^{\prime \prime}\right)$.

A weaker condition, restricted endowment additivity (Harless, 2017), applies only when no claimant is fully satisfied (when $f(c, E)<c$ in the definition of endowment additivity). These axioms can be criticized for rendering claims incommensurable with the endowment (Thomson, 2003). Only the proportional rule is endowment additive. However, restricted endowment additivity is satisfied by a broad class of rules. ${ }^{9}$

There are important differences between restricted endowment additivity and our additivity axioms. Our axioms require additivity in both the claims and endowment. The claims and the endowment are thus comparable under the same yardstick. Moreover, our axioms specify when two problems can be consolidated into a single problem; in contrast, restricted endowment additivity specifies when a claims problem can be split into two problems.

Restricted endowment additivity also has a dual:

Dual restricted endowment additivity: For each $(c, E) \in \mathcal{C}^{N}$ and each pair $E^{\prime}, E^{\prime \prime} \in \mathbb{R}_{+}$such that $E^{\prime}+E^{\prime \prime}=E+\sum_{N} c_{i}$, if $f(c, E)>0$, then $c+f(c, E)=$ $f\left(c, E^{\prime}\right)+f\left(c, E^{\prime \prime}\right)$.

The next axiom, multiplicative invariance, specifies that what is relevant in comparing two claims is not their size but only their ratio. Thus, multiplying all claims by the same constant should not affect the awards. Of course, this requires that the constant is such that, upon multiplying, the problem is still such that the sum of the claims is at least as large as the endowment.

\footnotetext{
${ }^{9}$ A rich sub-family, the "PW-proportional rules," is characterized on the basis of restricted endowment additivity, consistency, and endowment continuity (Theorem 2 in Harless, 2017). The PW-proportional rules include but are not restricted to the CEA rule and its asymmetric generalizations (Flores-Szwagrzak, 2015), the PAP rules, as well as the discontinuous LCF rule.
} 
Multiplicative invariance: For each $(c, E) \in \mathcal{C}^{N}$ and each $\lambda \in \mathbb{R}$ such that $\lambda \sum_{N} c_{i} \geq E, f(\lambda c, E)=f(c, E)$.

Multiplicative invariance can also be criticized on the grounds that it breaks the comparability of the claims with the endowment since it enables scaling claims while keeping the endowment unaltered. It is straightforward to check that only the proportional rule satisfies multiplicative invariance (Proposition 1 in Marchant, 2008). Our weaker version of the axiom only applies when all claimants make positive losses. As with multiplicative invariance, we need to specify the range of the scaling constant; we do so by specifying that all claims increase in the same proportion. ${ }^{10}$

Restricted multiplicative invariance: For each $(c, E) \in \mathcal{C}^{N}$ such that $f(c, E)<c$ and each scalar $\lambda>1, f(\lambda c, E)=f(c, E)$.

Restricted multiplicative invariance is satisfied by many rules beyond the proportional rule. The constrained equal awards rule, the priority-augmented weighted CEA rules (Flores-Szwagrzak, 2015), and the collectively rational solutions (Stovall, 2014b) satisfy this property.

Theorem 2. The PAP rules are the only bilaterally consistent and continuous rules satisfying any one of the following combinations of axioms:

(a) restricted additivity and restricted endowment additivity,

(b) restricted endowment additivity and dual restricted endowment additivity,

(c) restricted additivity and multiplicative invariance.

Theorem 2 can also be used to characterize the proportional rule in the same way Theorem 1 was used to characterize it in Corollary 1.

\section{Conclusion}

Priority and proportionality are two central principles in the theory of distributive justice (Young, 1994). In the context of the claims problem, their recommendations seem to be at odds: Either claimants are prioritized on the basis of their non-claim individual characteristics or the focus is solely on their claims, assigning the same award to each dollar claimed under proportionality. Real-life bankruptcy rules however combine priority and proportionality; though stylized, the priority-augmented proportional (PAP) rules studied here resemble them broadly.

\footnotetext{
${ }^{10}$ Alternatively, we could specify that, for each $(c, E) \in \mathcal{C}^{N}$ such that $f(c, E)<c$ and each $\lambda \in \mathbb{R}$ such that $f(c, E)<\lambda c, f(\lambda c, E)=f(c, E)$.
} 


\begin{tabular}{l|ccccc} 
& $P$ & $P^{\succsim}$ & $C E L$ & $C E A$ & $L C F$ \\
\hline consistency & $\checkmark$ & $\checkmark$ & $\checkmark$ & $\checkmark$ & $\checkmark$ \\
continuity & $\checkmark$ & $\checkmark$ & $\checkmark$ & $\checkmark$ & $x$ \\
restricted additivity & $\checkmark$ & $\checkmark$ & $\checkmark$ & $x$ & $\checkmark$ \\
dual restricted additivity & $\checkmark$ & $\checkmark$ & $x$ & $\checkmark$ & $\checkmark$ \\
endowment linearity & $\checkmark$ & $x$ & $x$ & $x$ & $x$ \\
endowment additivity & $\checkmark$ & $x$ & $x$ & $x$ & $x$ \\
restricted endowment additivity & $\checkmark$ & $\checkmark$ & $x$ & $\checkmark$ & $\checkmark$ \\
dual restricted endowment additivity & $\checkmark$ & $\checkmark$ & $\checkmark$ & $x$ & $\checkmark$ \\
restricted multiplicative invariance & $\checkmark$ & $\checkmark$ & $x$ & $\checkmark$ & $\checkmark$ \\
self-duality & $\checkmark$ & $x$ & $x$ & $x$ & $x$ \\
equal treatment of equal claims & $\checkmark$ & $x$ & $\checkmark$ & $\checkmark$ & $\checkmark$
\end{tabular}

Table 1: Note that $P \succsim$ refers to any PAP rule other than $P$. Recall that $L C F$ denotes the largerclaims-first rule introduced in Section 2.

This paper proposes two axioms satisfied by all PAP rules, restricted additivity and its dual axiom. Within the class of all consistent and continuous rules, only the PAP rules satisfy these two axioms (Theorem 1). We provide two additional characterizations replacing dual restricted additivity with restricted endowment additivity and restricted multiplicative invariance; a further characterization also replaces restricted additivity with the axiom dual to restricted endowment additivity (Theorem 2). Restricted endowment additivity and restricted multiplicative invariance are versions of stronger axioms only satisfied by the proportional rule.

A natural question is whether other axioms characterizing the proportional rule can be adapted to characterize the PAP rules. Unfortunately, the main characterizations of the proportional rule use axioms specifying that no claimant group should be able to gain by reallocating or transferring parts of their claims across themselves. ${ }^{11}$ These axioms cannot formulated naturally together with priorities.

Other additivity axioms identify a sub-class of claims problems where the rule to be characterized is additive. Examples of this approach include the additivity axioms used in the characterization of the Ibn Ezra rule (Bergantiños and MéndezNaya, 2001), the minimal overlap rule (Alcalde et al., 2014), and the reverse Talmud rules (Arin et al., 2017). The proportional rule is not additive in these specific domains; moreover, these additivity properties exclude rules that may prioritize certain claimants.

It is an open question if bilateral consistency can be replaced by other well known

\footnotetext{
${ }^{11}$ For example, see O’Neill (1982), de Frutos (1999), Moreno-Ternero (2006), and Ju et al. (2007).
} 
consistency axioms such as "null claims consistency," "null compensation consistency," or "full compensation consistency" in our characterizations. ${ }^{12}$ It may also be possible to weaken continuity to claims continuity. Finally, we might ask what properties lead to rules that replace the proportional rule with the CEA or CEL rules in our analysis. ${ }^{13}$

\section{References}

Alcalde, J., M. del Carmen Marco-Gil, and J. A. Silva-Reus (2014). The minimal overlap rule: restrictions on mergers for creditors' consensus. Top 22(1), 363-383.

Arin, J., J. Benito-Ostolaza, and E. Inarra (2017). The reverse talmud family of rules for banktruptcy problems: A characterization. Mathematical Social Sciences 89, 43-49.

Aumann, R. and M. Maschler (1985). Game theoretic analysis of a bankruptcy problem from the Talmud. Journal of Economic Theory 36(2), 195-213.

Bergantiños, G. and L. Méndez-Naya (2001). Additivity in bankruptcy problems and in allocation problems. Spanish Economic Review 3(3), 223-229.

Bergantiños, G. and J. J. Vidal-Puga (2004). Additive rules in bankruptcy problems and other related problems. Mathematical Social Sciences 47(1), 87-101.

Chun, Y. (1988). The proportional solution for rights problems. Mathematical Social Sciences 15(3), 231-246.

Chun, Y. (1999). Equivalence of axioms for bankruptcy problems. International Journal of Game Theory 28(4), 511-520.

Csoka, P. and J. Herings (2016). An axiomatization of the proportional rule in financial networks. Maastricht University Working paper.

de Frutos, M. (1999). Coalitional manipulation in a bankruptcy problem. Review of Economic Design 4 (3), 255-272.

Flores-Szwagrzak, K. (2015). Priority classes and weighted constrained equal awards rules for the claims problem. Journal of Economic Theory 160, 36 - 55.

Harless, P. (2017). Endowment additivity and the weighted proportional rules for adjudicating conflicting claims. Economic Theory 63(3), 755-781.

Hokari, T. and W. Thomson (2003). Claims problems and weighted generalizations of the Talmud rule. Economic Theory 21(2), 241-261.

Ju, B., E. Miyagawa, and T. Sakai (2007). Non-manipulable division rules in claim problems and generalizations. Journal of Economic Theory 132, 1-26.

\footnotetext{
${ }^{12}$ See Chapter 10 in Thomson (2019) for a detailed exposition of these axioms.

${ }^{13}$ Hokari and Thomson (2003) tackle a similar question for the Talmud rule.
} 
Marchant, T. (2008). Scale invariance and similar invariance conditions for bankruptcy problems. Social Choice and Welfare 31(4), 693-707.

Moreno-Ternero, J. (2006). Proportionality and non-manipulability in bankruptcy problems. International Game Theory Review 8(01), 127-139.

Moulin, H. (1987). Equal or proportional division of a surplus, and other methods. International Journal of Game Theory 16(3), 161-186.

Moulin, H. (2000). Priority rules and other asymmetric rationing methods. Econometrica 68(3), 643-684.

Moulin, H. (2002). Axiomatic cost and surplus-sharing. In K. Arrow, A. Sen, and K. Suzumura (Eds.), Handbook of Social Choice and Welfare, Volume 1, pp. 289357. Elsevier.

O'Neill, B. (1982). A problem of rights arbitration from the Talmud. Mathematical Social Sciences 2(4), 345 - 371.

Roth, A. E. (1988). The Shapley value: essays in honor of Lloyd S. Shapley. Cambridge University Press. New York, NY, USA.

Shapley, L. (1953). A value for $n$-person games. Annals of Mathematical Studies 28, $307-317$.

Stovall, J. (2014a). Asymmetric parametric division rules. Games and Economic Behavior 84(1), $87-110$.

Stovall, J. (2014b). Collective rationality and monotone path division rules. Journal of Economic Theory 154, 1-24.

Thomson, W. (2003). Axiomatic and game-theoretic analysis of bankruptcy and taxation problems: a survey. Mathematical Social Sciences 45(3), 249 - 297.

Thomson, W. (2011). Consistency and its converse: an introduction. Review of Economic Design 15(4), 257 - 291.

Thomson, W. (2015). Axiomatic and game-theoretic analysis of bankruptcy and taxation problems: an update. Mathematical Social Sciences 74, 41-59.

Thomson, W. (2016). A new characterization of the proportional rule for claims problems. Economics Letters 154, 255-257.

Thomson, W. (2019). How to Divide When There Isn't Enough: From Aristotle, the Talmud, and Maimonides to the Axiomatics of Resource Allocation. Cambridge University Press.

Young, P. (1987). On dividing an amount according to claims or liabilities. Mathematics of Operations Research 12(3), 397 - 414.

Young, P. (1988). Distributive justice in taxation. Journal of Economic Theory $44(2), 321-335$.

Young, P. (1994). Equity: In Theory and Practice. Princeton University Press. 


\section{A Appendix}

\section{A.1 Preliminary observations}

The PAP rules satisfy two axioms that will be important in our proofs. The classical endowment monotonicity axiom requires that all awards move in the same direction in response to a change in the endowment.

Endowment monotonicity: For each $(c, E) \in \mathcal{C}^{N}$ and each $E^{\prime} \in[0, E], f\left(c, E^{\prime}\right) \leq$ $f(c, E)$.

Converse consistency requires that if an allocation is such that its restriction is chosen by the rule for each two-claimant subgroup, then the allocation is chosen by the rule for the whole group.

Converse consistency: For each $N \in \mathcal{N}$ and each $(c, E) \in \mathcal{C}^{N}$,

$\left[x \in Z(c, E)\right.$ and, for each $\left.\{i, j\} \subseteq N, f\left(c_{\{i, j\}}, x_{i}+x_{j}\right)=x_{\{i, j\}}\right]$ implies $f(c, E)=x$.

Lemma 1. The PAP rules are consistent, continuous and satisfy restricted additivity, dual restricted additivity, converse consistency, restricted endowment additivity, dual restricted endowment additivity, restricted multiplicative invariance, and endowment monotonicity.

Proof. The PAP rules belong to the wider family of consistent, continuous, and endowment monotonic rules characterized by Moulin (2000). The PAP rules also belong to the PW-proportional rules characterized, by among other axioms, restricted endowment additivity (see Theorem 2 in Harless, 2017). An endowment monotonic and consistent rule is conversely consistent; Chun (1999) proves that endowment monotonicity and consistency imply an even stronger converse consistency property. Thus, each PAP rule is conversely consistent.

It remains to show that the each PAP rule satisfies restricted additivity, dual restricted additivity, and restricted multiplicative invariance. Let $\succsim$ denote a priority relation over $\mathbb{N}$ and let $N_{1}, N_{2}, \ldots, N_{k}$ denote the partition of $N$ induced by $\succsim$.

$P^{\succsim}$ satisfies restricted additivity. Let $c \in \mathbb{R}^{N}$ and $E, E^{\prime} \in\left[0, \sum_{N} c_{i}\right]$ be such that $P^{\succsim}(c, E)>0$, and $P^{\succsim}\left(c, E^{\prime}\right)>0$. By the definition of $P^{\succsim}$, since $P^{\succsim}(c, E)>0$, and $P^{\succsim}\left(c, E^{\prime}\right)>0$

$$
\text { for each } i \in N \backslash N_{k}, \quad P_{i}^{\succsim}(c, E)=c_{i} \quad \text { and } \quad P_{i}^{\succsim}\left(c, E^{\prime}\right)=c_{i},
$$

and, letting $\tilde{E}=E-\sum_{N \backslash N_{k}} c_{j}$ and $\tilde{E}^{\prime}=E^{\prime}-\sum_{N \backslash N_{k}} c_{j}$,

$$
\text { for each } i \in N_{k}, \quad P_{i}^{\succsim}(c, E)=P_{i}\left(c_{N_{k}}, \tilde{E}\right) \quad \text { and } \quad P_{i}^{\succsim}\left(c, E^{\prime}\right)=P_{i}\left(c_{N_{k}}, \tilde{E}^{\prime}\right) \text {. }
$$


Thus, by (1),

$$
\text { for each } i \in N \backslash N_{k}, \quad P_{i}^{\succsim}(c, E)+P_{i}^{\succsim}\left(c, E^{\prime}\right)=2 c_{i} \text {. }
$$

and, by (2),

$$
\begin{aligned}
& \text { for each } i \in N_{k}, \quad P_{i}^{\succsim}(c, E)+P_{i}^{\succsim}\left(c, E^{\prime}\right)=\frac{\tilde{E}}{\sum_{N_{k}} c_{j}} c_{i}+\frac{\tilde{E}^{\prime}}{\sum_{N_{k}} c_{j}} c_{i} \\
& =\frac{\tilde{E}+\tilde{E}^{\prime}}{\sum_{N_{k}} c_{j}} c_{i}=\frac{\tilde{E}+\tilde{E}^{\prime}}{2 \sum_{N_{k}} c_{j}} 2 c_{i} \\
& =P_{i}\left(2 c_{N_{k}}, \tilde{E}+\tilde{E}^{\prime}\right) \text {. }
\end{aligned}
$$

By (3), E+ $E^{\prime} \geq 2 \sum_{N \backslash N_{k}} c_{j}$. Thus, by the definition of $P^{\succsim}$,

$$
\text { for each } i \in N \backslash N_{k}, \quad P_{i}^{\succsim}\left(2 c, E+E^{\prime}\right)=P_{i}^{\succsim}\left(2 c, E+E^{\prime}\right)=2 c_{i} \text {. }
$$

Thus, the excess $E+E^{\prime}-2 \sum_{N \backslash N_{k}} c_{j}=\tilde{E}+\tilde{E}^{\prime}$ is distributed by $P^{\succsim}$ among the lowest priority class $N_{k}$ using the proportional rule,

$$
\text { for each } i \in N_{k}, \quad P_{i}^{\succsim}\left(2 c, E+E^{\prime}\right)=P_{i}\left(2 c_{N_{k}}, \tilde{E}+\tilde{E}^{\prime}\right) \text {. }
$$

Comparing (3) and (4) to (5) and (6), it follows that $P^{\succsim}\left(2 c, E+E^{\prime}\right)=P^{\succsim}(c, E)+$ $P^{\succsim}\left(c, E^{\prime}\right)$. The proof that each PAP rule satisfies dual restricted additivity is analogous.

$P^{\succsim}$ satisfies multiplicative invariance. Let $(c, E) \in \mathcal{C}^{N}$ be such that $P^{\succsim}(c, E)<c$ and $\lambda>1$. By the definition of a PAP rule, $P^{\succsim}(c, E)<c$ implies $E<\sum_{N_{1}} c_{j}$, and

(i) for each $i \in N_{1}, P_{i}^{\succsim}(c, E)=P_{i}\left(c_{N_{h}}, E\right)$,

(ii) for each $t>1$ and each $i \in N_{t}, P_{i}^{\succsim}(c, E)=0$.

Since $E<\sum_{N_{1}} c_{j}<\sum_{N_{1}} \lambda c_{j}$, using the definition of a PAP rule again as well as (i) and (ii) above,

(i') for each $i \in N_{1}, P_{i}^{\succsim}(\lambda c, E)=P_{i}\left(\lambda c_{N_{1}}, E\right)=P_{i}\left(c_{N_{1}}, E\right)$, and

(ii') for each $t>1$ and each $i \in N_{t}, P_{i}^{\succsim}(\lambda c, E)=0$.

Thus, $f(c, E)=f(\lambda c, E)$. 
Lemma 2. Suppose that $f$ satisfies continuity, restricted additivity, and either dual restricted additivity or restricted multiplicative invariance. Then, $f$ is endowment monotonic for claims problems involving two claimants.

Proof. Let $f$ satisfy continuity and restricted additivity. Let $\{i, j\}=N \in \mathcal{N}$, $(c, E) \in \mathcal{C}^{N}, E^{\prime} \in\left(E, c_{i}+c_{j}\right], x=f(c, E)$, and $x^{\prime}=f\left(c, E^{\prime}\right)$.

We will prove that $x^{\prime} \geq x$. By way of contradiction, suppose that $x_{i}^{\prime}<x_{i}$ and $x_{j}<x_{j}^{\prime}$. Let $y_{i}=x_{i}^{\prime}+\frac{1}{3}\left(x_{i}-x_{i}^{\prime}\right)$ and $z_{i}=x_{i}^{\prime}+\frac{2}{3}\left(x_{i}-x_{i}^{\prime}\right)$. Since $x_{i}^{\prime}<x_{i}$,

$$
0 \leq x_{i}^{\prime}<y_{i}<z_{i}<x_{i} \leq c_{i} .
$$

Let $F:[0,1] \rightarrow \mathbb{R}_{+}$be such that, for each $\lambda \in[0,1], F(\lambda)=f_{i}\left(c, \lambda E^{\prime}+(1-\lambda) E\right)$. By the continuity of $f, F$ is continuous. Thus, by the Intermediate Value Theorem and because $F(1)=x_{i}^{\prime}<y_{i}<x_{i}=F(0)$, there is a $\lambda_{y} \in(0,1)$ such that $F\left(\lambda_{y}\right)=y_{i}$. Let $e_{y}=\lambda_{y} E^{\prime}+\left(1-\lambda_{y}\right) E$ and $y_{j}=e_{y}-y_{i}$. By definition, $F\left(\lambda_{y}\right)=y_{i}<z_{i}<x_{i}=$ $F(0)$. Thus, by the Intermediate Value Theorem, there is a $\lambda_{z} \in\left(0, \lambda_{y}\right)$ such that $F\left(\lambda_{z}\right)=z_{i}$. Let $e_{z}=\lambda_{z} E^{\prime}+\left(1-\lambda_{z}\right) E$ and $z_{j}=e_{z}-z_{i}$.

Since $0<\lambda_{z}<\lambda_{y}<1, E<e_{z}<e_{y}<E^{\prime}$. Since $z_{i}+z_{j}=e_{z}<e_{y}=y_{i}+y_{j}$ and $y_{i}<z_{i}, z_{j}<y_{j}$. Since $x_{i}+x_{j}=E<e_{z}=z_{i}+z_{j}$ and $z_{i}<x_{i}, x_{j}<z_{j}$. Since $y_{i}+y_{j}=e_{y}<E^{\prime}=x_{i}^{\prime}+x_{j}^{\prime}$ and $x_{i}^{\prime}<y_{i}, y_{j}<x_{j}^{\prime}$. Altogether,

$$
0 \leq x_{j}<z_{j}<y_{j}<x_{j}^{\prime} \leq c_{j} .
$$

Let $y=\left(y_{i}, y_{j}\right)=f\left(c, e_{y}\right)$ and $z=\left(z_{i}, z_{j}\right)=f\left(c, e_{z}\right)$. Note that $0<y=f\left(c, e_{y}\right)<c$ and $0<z=f\left(c, e_{z}\right)<c$.

Suppose that $f$ satisfies dual restricted additivity. We distinguish the four possible cases and arrive at a contradiction in each:

Case 1: $f\left(c, e_{y}-e_{z}\right)<c$. By dual restricted additivity,

$$
f\left(c, e_{y}\right)=f\left(c, e_{y}\right)+f(c, 0)=f\left(2 c, e_{y}\right)=f\left(c, e_{z}\right)+f\left(c, e_{y}-e_{z}\right) \geq f\left(c, e_{z}\right) .
$$

This contradicts the fact that $y_{i}<z_{i}$.

Case 2: $f\left(c, e_{y}-e_{z}\right)>0$. By restricted additivity, $f\left(2 c, e_{y}\right)=f\left(c, e_{y}-e_{z}\right)+f\left(c, e_{z}\right)$. Since $f\left(c, e_{y}\right)<c$, dual restricted additivity implies that $f\left(2 c, e_{y}\right)=f\left(c, e_{y}\right)+$ $f(c, 0)=f\left(c, e_{y}\right)$. Thus,

$$
f\left(c, e_{y}\right)=f\left(2 c, e_{y}\right)=f\left(c, e_{y}-e_{z}\right)+f\left(c, e_{z}\right) \geq f\left(c, e_{z}\right) .
$$

This contradicts the fact that $y_{i}<z_{i}$. 
Case 3: $f_{i}\left(c, e_{y}-e_{z}\right)=c_{i}$ and $f_{j}\left(c, e_{y}-e_{z}\right)=0$. By continuity, there is an $\epsilon>0$ such that $0<f\left(c, e_{y}+\epsilon\right)<c, f\left(c, e_{y}-e_{z}+\epsilon\right)>0$, and $z_{i}>f_{i}\left(c, e_{y}+\epsilon\right)$. By dual restricted additivity, $f\left(c, e_{y}+\epsilon\right)=f\left(c, e_{y}+\epsilon\right)+f(c, 0)=f\left(2 c, e_{y}+\epsilon\right)$. By restricted additivity,

$$
f\left(2 c, e_{y}+\epsilon\right)=f\left(c, e_{y}-e_{z}+\epsilon\right)+f\left(c, e_{z}\right) .
$$

Thus, $f\left(c, e_{y}+\epsilon\right)=f\left(2 c, e_{y}+\epsilon\right)=f\left(c, e_{y}-e_{z}+\epsilon\right)+f\left(c, e_{z}\right) \geq f\left(c, e_{z}\right)$. Since $z=f\left(c, e_{z}\right)$, this contradicts the fact that $z_{i}>f_{i}\left(c, e_{y}+\epsilon\right)$.

Case 4: $f_{i}\left(c, e_{y}-e_{z}\right)=0$ and $f_{j}\left(c, e_{y}-e_{z}\right)=c_{j}$. Exchanging the roles of $i$ and $j$ in the previous case, we again arrive at a contradiction.

Suppose that $f$ satisfies restricted multiplicative invariance. We distinguish the four possible cases and arrive at a contradiction in each:

Case 1: $f\left(c, e_{y}-e_{z}\right)<c$. By restricted multiplicative invariance, $f\left(c, e_{y}\right)=f\left(2 c, e_{y}\right)$. By restricted additivity,

$$
f\left(2 c, e_{y}\right)=f\left(c, e_{z}\right)+f\left(c, e_{y}-e_{z}\right) \geq f\left(c, e_{z}\right) .
$$

Thus, $f\left(c, e_{y}\right) \geq f\left(c, e_{z}\right)$. This contradicts the fact that $y_{i}<z_{i}$.

Case 2: $f\left(c, e_{y}-e_{z}\right)>0$. By restricted additivity, $f\left(2 c, e_{y}\right)=f\left(c, e_{y}-e_{z}\right)+f\left(c, e_{z}\right)$. Since $f\left(c, e_{y}\right)<c$, restricted multiplicative invariance implies that $f\left(2 c, e_{y}\right)=f\left(c, e_{y}\right)$. Thus,

$$
f\left(c, e_{y}\right)=f\left(2 c, e_{y}\right)=f\left(c, e_{y}-e_{z}\right)+f\left(c, e_{z}\right) \geq f\left(c, e_{z}\right) .
$$

This contradicts the fact that $y_{i}<z_{i}$.

Case 3: $f_{i}\left(c, e_{y}-e_{z}\right)=c_{i}$ and $f_{j}\left(c, e_{y}-e_{z}\right)=0$. By continuity, there is an $\epsilon>0$ such that $0<f\left(c, e_{y}+\epsilon\right)<c, f\left(c, e_{y}-e_{z}+\epsilon\right)>0$, and $z_{i}>f_{i}\left(c, e_{y}+\epsilon\right)$. By restricted multiplicative invariance, $f\left(c, e_{y}+\epsilon\right)=f\left(2 c, e_{y}+\epsilon\right)$. By restricted additivity,

$$
f\left(2 c, e_{y}+\epsilon\right)=f\left(c, e_{y}-e_{z}+\epsilon\right)+f\left(c, e_{z}\right) .
$$

Thus, $f\left(c, e_{y}+\epsilon\right)=f\left(2 c, e_{y}+\epsilon\right)=f\left(c, e_{y}-e_{z}+\epsilon\right)+f\left(c, e_{z}\right) \geq f\left(c, e_{z}\right)$. Since $z=f\left(c, e_{z}\right)$, this contradicts the fact that $z_{i}>f_{i}\left(c, e_{y}+\epsilon\right)$.

Case 4: $f_{i}\left(c, e_{y}-e_{z}\right)=0$ and $f_{j}\left(c, e_{y}-e_{z}\right)=c_{j}$. Exchanging the roles of $i$ and $j$ in the previous case, we again arrive at a contradiction. 


\section{A.2 Proof of Theorem 1}

Lemma 3. Let $f$ denote a continuous rule.

If $f$ satisfies restricted additivity, then, for each $(c, E) \in \mathcal{C}^{N}$ and $E^{\prime} \in\left(E, \sum_{N} c_{i}\right]$,

$\left[\forall \lambda \in[0,1], f\left(c, \lambda E^{\prime}+(1-\lambda) E\right)>0\right] \Rightarrow f\left(c, \lambda E^{\prime}+(1-\lambda) E\right)=\lambda f\left(c, E^{\prime}\right)+(1-\lambda) f(c, E)$.

If $f$ satisfies dual restricted additivity, then, for each $(c, E) \in \mathcal{C}^{N}$ and $E^{\prime} \in\left(E, \sum_{N} c_{i}\right]$,

$\left[\forall \lambda \in[0,1], f\left(c, \lambda E^{\prime}+(1-\lambda) E\right)<c\right] \Rightarrow f\left(c, \lambda E^{\prime}+(1-\lambda) E\right)=\lambda f\left(c, E^{\prime}\right)+(1-\lambda) f(c, E)$.

Proof. We only prove the first statement; the proof of the second statement is analogous. Let $f$ denote a continuous rule satisfying restricted additivity. Let $(c, E) \in \mathcal{C}^{N}$ and $E^{\prime} \in\left(E, \sum_{N} c_{i}\right]$ be such that,

$$
\text { for each } \lambda \in[0,1], f\left(c, \lambda E^{\prime}+(1-\lambda) E\right)>0 \text {. }
$$

Let $i \in N, e, e^{\prime} \in\left[E, E^{\prime}\right]$, and $e^{\prime \prime}=\frac{1}{2}\left(e+e^{\prime}\right)$. Since $\left[E, E^{\prime}\right]$ is convex, $e^{\prime \prime} \in\left[E, E^{\prime}\right]$. Thus, there are $\lambda, \lambda^{\prime}, \lambda^{\prime \prime} \in[0,1]$ such that

$$
\lambda E^{\prime}+(1-\lambda) E=e, \lambda^{\prime} E^{\prime}+\left(1-\lambda^{\prime}\right) E=e^{\prime} \text {, and } \lambda^{\prime \prime} E^{\prime}+\left(1-\lambda^{\prime \prime}\right) E=e^{\prime \prime} .
$$

Thus, by $(7), f_{i}(c, e)>0, f_{i}\left(c, e^{\prime}\right)>0$, and $f_{i}\left(c, e^{\prime \prime}\right)>0$. Thus, by restricted additivity, $f_{i}\left(2 c, 2 e^{\prime \prime}\right)=2 f_{i}\left(c, e^{\prime \prime}\right)$ and $f_{i}\left(2 c, e+e^{\prime}\right)=f_{i}(c, e)+f_{i}\left(c, e^{\prime}\right)$. By the definition of $e^{\prime \prime}, 2 e^{\prime \prime}=e+e^{\prime}$. Thus, $2 f_{i}\left(c, e^{\prime \prime}\right)=f_{i}\left(2 c, e+e^{\prime}\right)=f_{i}(c, e)+f_{i}\left(c, e^{\prime}\right)$. By the definition of $e^{\prime \prime}, 2 f_{i}\left(c, \frac{1}{2} e+\frac{1}{2} e^{\prime}\right)=f_{i}(c, e)+f_{i}\left(c, e^{\prime}\right)$. Thus, we have proven that,

$$
\text { for each pair } e, e^{\prime} \in\left[E, E^{\prime}\right], f_{i}\left(c, \frac{e+e^{\prime}}{2}\right)=\frac{f_{i}(c, e)+f_{i}\left(c, e^{\prime}\right)}{2} \text {. }
$$

Let $g:\left[E, E^{\prime}\right] \rightarrow \mathbb{R}$ be defined by $g(e)=f_{i}(c, e)$ for each $e \in\left[E, E^{\prime}\right]$. By $(8), g$ is midpoint convex on $\left[E, E^{\prime}\right]$. Moreover, since $f$ is continuous, $g$ is continuous as well. Thus, $g$ is convex on $\left[E, E^{\prime}\right]$. Thus, by (8), for each $\lambda \in[0,1], g\left(\lambda E^{\prime}+(1-\lambda) E\right)=$ $\lambda g\left(E^{\prime}\right)+(1-\lambda) g(E)$. Thus, for each $\lambda \in[0,1]$

$$
f_{i}\left(c, \lambda E^{\prime}+(1-\lambda) E\right)=\lambda f_{i}\left(c, E^{\prime}\right)+(1-\lambda) f_{i}(c, E) .
$$

Repeating the above argument for each $i \in N$, for each $\lambda \in[0,1], f\left(c, \lambda E^{\prime}+(1-\right.$ $\lambda) E)=\lambda f\left(c, E^{\prime}\right)+(1-\lambda) f(c, E)$. 
For each pair $i, j \in \mathbb{N}$ and each $(c, E) \in \mathcal{C}^{\{i, j\}}$, let $D^{i j}(c, E) \in \mathbb{R}^{\{i, j\}}$ denote the allocation fully prioritizing claimant $i$ over claimant $j$,

$$
D_{i}^{i j}(c, E)=\min \left\{c_{i}, E\right\} \quad \text { and } \quad D_{j}^{i j}(c, E)=\max \left\{0, E-c_{i}\right\} .
$$

The following lemma shows that, if a rule satisfies continuity, restricted additivity, and dual restricted additivity, then its recommended allocations in two-claimant problems involving claimants $i$ and $j$ coincide with those specified by $P, D^{i j}$, or $D^{j i}$, as illustrated in Figure 1.

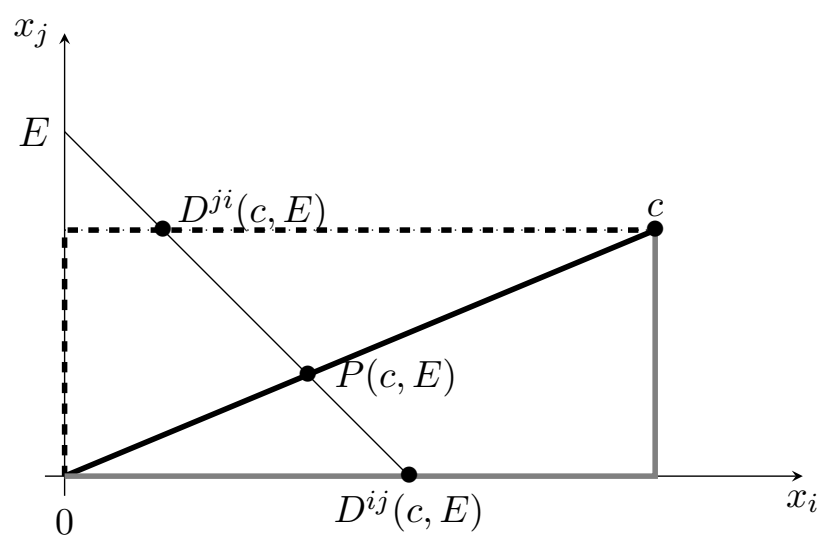

Figure 1: A claims problem $(c, E)$ involving claimants $i$ and $j$. The $x_{i}$ axis measures the award of claimant $i$ and the $x_{j}$ axis measures the award of claimant $j$. As $E$ increases from 0 to $c_{i}+c_{j}$, the allocations recommended by a continuous rule satisfying restricted additivity and dual restricted additivity will follow one of the three paths (dashed, solid, gray) connecting the origin and $c$.

Lemma 4. Let $f$ denote a continuous rule satisfying restricted additivity and dual restricted additivity. Let $D^{i j}$ and $D^{j i}$ be as defined in (9). Then, for each $\{i, j\} \subseteq \mathbb{N}$, $f$ coincides with $P, D^{i j}$, or $D^{j i}$ for claims problems in $\mathcal{C}^{\{i, j\}}$.

Proof. Let $f$ denote a rule satisfying the axioms in Lemma 4 . By Lemma $2, f$ is endowment monotonic for two-claimant problems, a fact we will use repeatedly below. Let $N \in \mathcal{N}$ consist of two claimants. Without loss of generality, let $N=\{1,2\}$.

Claim 1. If $(c, E) \in \mathcal{C}^{N}$ is such that $0<f(c, E)<c$, then $f(c, E)=P(c, E)$.

Let $(c, E) \in \mathcal{C}^{N}$ be such that $0<f(c, E)<c$. Let $\lambda \in[0,1]$ and $e^{*}=\lambda E+(1-\lambda) 0$. Since $f(c, E)<c$ and $f(c, 0)=0<c$, by Lemma 3 ,

$$
f\left(c, e^{*}\right)=f(c, \lambda E)=\lambda f(c, E)
$$


Since $f(c, E)>0$, then $f\left(c, e^{*}\right)=\lambda f(c, E)>0$. By endowment monotonicity, if $e^{1} \geq E$, then $f\left(c, e^{1}\right) \geq f(c, E)>0$. Let $\beta \in(0,1)$. Given that $f\left(c, \lambda\left(c_{1}+c_{2}\right)+(1-\right.$ $\lambda) \beta E)>0$, by Lemma $3, f\left(c, \lambda\left(c_{1}+c_{2}\right)+(1-\lambda) \beta E\right)=\lambda f\left(c, c_{1}+c_{2}\right)+(1-\lambda) f(c, \beta E)$. By endowment monotonicity, $f(c, \cdot)$ is continuous. Taking limits as $\beta \rightarrow 0$,

$$
f\left(c, \lambda\left(c_{1}+c_{2}\right)+(1-\lambda) 0\right)=\lambda f\left(c, c_{1}+c_{2}\right)+(1-\lambda) f(c, 0)
$$

Thus, for each $\lambda \in[0,1], f\left(c, \lambda\left(c_{1}+c_{2}\right)\right)=\lambda f\left(c, c_{1}+c_{2}\right)$. Note that $f(c, E)>0$ implies $c>0$. Letting $\lambda^{*}=\frac{E}{c_{1}+c_{2}}, f\left(c, \lambda^{*}\left(c_{1}+c_{2}\right)\right)=\lambda^{*} f\left(c, c_{1}+c_{2}\right)=\lambda^{*} c$. Thus, $f(c, E)=\frac{E}{c_{1}+c_{2}} c=P(c, E)$.

Claim 2. If $(c, E) \in \mathcal{C}^{N}$ is such that $0<f(c, E)<c$ is not true, then either $f(c, E)=D^{12}(c, E)$ or $f(c, E)=D^{21}(c, E)$.

If $(c, E) \in \mathcal{C}^{N}$ is such that $0<f(c, E)<c$ is not true then, either $f_{1}(c, E)=$ $\min \left\{c_{1}, E\right\}$ or $f_{2}(c, E)=\min \left\{c_{2}, E\right\}$.

Let $\mathcal{D} \subseteq \mathcal{C}^{N}$ consist of all $(c, E) \in \mathcal{C}^{N}$ such that $c>0$ and $0<E<c_{1}+c_{2}$. Note that all rules coincide for claims problems in $\mathcal{C}^{N} \backslash \mathcal{D}$.

Claim 3. Let $(c, E) \in \mathcal{D}$.

(i) If $f(c, E)=P(c, E)$, then, for each $\left(c^{\prime}, E^{\prime}\right) \in \mathcal{C}^{N}, f\left(c^{\prime}, E^{\prime}\right)=P\left(c^{\prime}, E^{\prime}\right)$.

(ii) If $f(c, E)=D^{12}(c, E)$, then, for each $\left(c^{\prime}, E^{\prime}\right) \in \mathcal{C}^{N}, f\left(c^{\prime}, E^{\prime}\right)=D^{12}\left(c^{\prime}, E^{\prime}\right)$.

(iii) If $f(c, E)=D^{21}(c, E)$ then, for each $\left(c^{\prime}, E^{\prime}\right) \in \mathcal{C}^{N}, f\left(c^{\prime}, E^{\prime}\right)=D^{21}\left(c^{\prime}, E^{\prime}\right)$.

We prove statement (i); the proofs of (ii) and (iii) are analogous. Let $(c, E) \in \mathcal{D}$ and suppose that $f(c, E)=P(c, E)$. Suppose, by way of contradiction, that there is $\left(c^{\prime}, E^{\prime}\right) \in \mathcal{C}^{N}$ such that $f\left(c^{\prime}, E^{\prime}\right) \neq P\left(c^{\prime}, E^{\prime}\right)$ which requires that $\left(c^{\prime}, E^{\prime}\right) \in \mathcal{D}$. For each $\lambda \in[0,1]$, let

$$
\left(c^{\lambda}, e^{\lambda}\right)=\lambda\left(c^{\prime}, E^{\prime}\right)+(1-\lambda)(c, E) \quad \text { and } \quad G(\lambda)=f_{1}\left(c^{\lambda}, e^{\lambda}\right)-P_{1}\left(c^{\lambda}, e^{\lambda}\right) .
$$

Since $f$ and $P$ are continuous, $G:[0,1] \rightarrow \mathbb{R}$ is a continuous function and, from its definition, $G(0)=0$ and $G(1) \neq 0$. Let $\mu=\sup \{\lambda \in[0,1]: G(\lambda)=0\}$. By continuity, $G(\mu)=0$. Thus, $\mu<1$. Consider a sequence $\left\{\lambda_{k}\right\}$ converging to $\mu$ and such that, for each $k \in \mathbb{N}, \lambda_{k} \in(\mu, 1]$. By Claims 1 and 2, we can further select the members of the sequence so that at least one of the following cases holds:

Case 1: For each $k \in \mathbb{N}, f_{1}\left(c^{\lambda_{k}}, e^{\lambda_{k}}\right)=D_{1}^{12}\left(c^{\lambda_{k}}, e^{\lambda_{k}}\right)$. 
Case 2: For each $k \in \mathbb{N}, f_{1}\left(c^{\lambda_{k}}, e^{\lambda_{k}}\right)=D_{1}^{21}\left(c^{\lambda_{k}}, e^{\lambda_{k}}\right)$.

In Case 1 , by the continuity of $D^{12}$ and $P$,

$$
G\left(\lambda_{k}\right) \stackrel{k \rightarrow \infty}{\rightarrow} D_{1}^{12}\left(c^{\mu}, e^{\mu}\right)-P\left(c^{\mu}, e^{\mu}\right)=\min \left\{c_{1}^{\mu}, e^{\mu}\right\}-\frac{e^{\mu}}{c_{1}^{\mu}+c_{2}^{\mu}} c_{1}^{\mu} \neq 0 .
$$

In Case 2, by the continuity of $D^{21}$ and $P$,

$$
G\left(\lambda_{k}\right) \stackrel{k \rightarrow \infty}{\rightarrow} D_{1}^{21}\left(c^{\mu}, e^{\mu}\right)-P\left(c^{\mu}, e^{\mu}\right)=\max \left\{0, e^{\mu}-c_{2}^{\mu}\right\}-\frac{e^{\mu}}{c_{1}^{\mu}+c_{2}^{\mu}} c_{1}^{\mu} \neq 0 .
$$

However, since $G$ is continuous, $\left\{\lambda_{k}\right\}$ converges to $\mu$, and $G(\mu)=0$, a contradiction. Thus, $f_{1}\left(c^{\prime}, E^{\prime}\right)=P\left(c^{\prime}, E^{\prime}\right)$.

Taken together, Claims 1, 2, and 3 complete the proof.

Proof of Theorem 1. By Lemma 1, each PAP rule satisfies bilateral consistency, continuity, restricted additivity, and dual restricted additivity. Conversely, let $f$ denote a rule satisfying these axioms. By Lemma 4 we can define a complete binary relation $\succsim$ over $\mathbb{N}$ from $f$ as follows: for each pair $i, j \in \mathbb{N}$,

$$
\begin{aligned}
& i \succ j \quad \Leftrightarrow \quad \text { for each }(c, E) \in \mathcal{C}^{\{i, j\}}, \quad f(c, E)=D^{i j}(c, E), \\
& i \sim j \quad \Leftrightarrow \quad \text { for each }(c, E) \in \mathcal{C}^{\{i, j\}}, \quad f(c, E)=P(c, E), \\
& i \succsim j \quad \Leftrightarrow \quad i \succ j \quad \text { or } i \sim j .
\end{aligned}
$$

By bilateral consistency, $\succsim$ is transitive. ${ }^{14}$ We now prove that $f=P \succsim$. Let $N \in \mathcal{N}$, $(c, E) \in \mathcal{C}^{N}$, and $x=f(c, E)$. By consistency,

$$
\text { for each pair } i, j \in N, \quad f\left(c_{\{i, j\}}, x_{i}+x_{j}\right)=x_{\{i, j\}} \text {. }
$$

By Lemma 4, for each pair $i, j \in N$, one of the following equations holds:

$$
\begin{aligned}
& f\left(c_{\{i, j\}}, x_{i}+x_{j}\right)=P\left(c_{\{i, j\}}, x_{i}+x_{j}\right), \\
& f\left(c_{\{i, j\}}, x_{i}+x_{j}\right)=D^{i j}\left(c_{\{i, j\}}, x_{i}+x_{j}\right), \\
& f\left(c_{\{i, j\}}, x_{i}+x_{j}\right)=D^{j i}\left(c_{\{i, j\}}, x_{i}+x_{j}\right) .
\end{aligned}
$$

\footnotetext{
${ }^{14}$ Let $i, j, k \in \mathbb{N}$ be such that $i \succsim j \succsim k$. We need to show that $i \succsim k$. Suppose, by way of contradiction, that $k \succ i$. Let $(c, E) \in \mathcal{C}^{\{i, j, k\}}$ be such that $c_{i}=c_{j}=c_{k}=E=1$ and let $x=f(c, E)$. By bilateral consistency, $x_{\{i, j\}}=f\left(c_{\{i, j\}}, x_{i}+x_{j}\right), x_{\{i, k\}}=f\left(c_{\{i, k\}}, x_{i}+x_{k}\right)$, and $x_{\{j, k\}}=f\left(c_{\{j, k\}}, x_{j}+x_{k}\right)$. Using (10), $k \succ i$ implies $x_{i}=0 ; j \succsim k$ implies $x_{j} \geq x_{k} ;$ and, $i \succsim j$ implies $x_{i} \geq x_{j}$. Thus, $x_{i}+x_{j}+x_{k}=1$ implies $x_{j}+x_{k}=1$. Thus, $x_{j} \geq \frac{1}{2}$. Thus, $x_{i} \geq x_{j}>0$, a contradiction. Thus, in fact, $i \succsim k$. Thus, $\succsim$ is transitive.
} 
By (10), for each pair $i, j \in N$,

$$
\begin{aligned}
& f\left(c_{\{i, j\}}, x_{i}+x_{j}\right)=P\left(c_{\{i, j\}}, x_{i}+x_{j}\right) \Rightarrow i \sim j, \\
& f\left(c_{\{i, j\}}, x_{i}+x_{j}\right)=D^{i j}\left(c_{\{i, j\}}, x_{i}+x_{j}\right) \Rightarrow i \succ j, \\
& f\left(c_{\{i, j\}}, x_{i}+x_{j}\right)=D^{j i}\left(c_{\{i, j\}}, x_{i}+x_{j}\right) \Rightarrow j \succ i .
\end{aligned}
$$

By the definition of $P^{\succsim}$, for each pair $i, j \in N$,

$$
\begin{aligned}
& i \sim j \Rightarrow P^{\succsim}\left(c_{\{i, j\}}, x_{i}+x_{j}\right)=P\left(c_{\{i, j\}}, x_{i}+x_{j}\right), \\
& i \succ j \Rightarrow P^{\succsim}\left(c_{\{i, j\}}, x_{i}+x_{j}\right)=D^{i j}\left(c_{\{i, j\}}, x_{i}+x_{j}\right), \\
& j \succ i \Rightarrow P^{\succsim}\left(c_{\{i, j\}}, x_{i}+x_{j}\right)=D^{j i}\left(c_{\{i, j\}}, x_{i}+x_{j}\right) .
\end{aligned}
$$

Since $\succsim$ is complete, for each pair $i, j \in N$, we have three possibilities: $i \sim j, i \succ j$, and $j \succ i$. Thus, by (11), (12), (13), and (14),

$$
\text { for each pair } i, j \in N, \quad P^{\succsim}\left(c_{\{i, j\}}, x_{i}+x_{j}\right)=x_{\{i, j\}} \text {. }
$$

By Lemma $1, P^{\succsim}$ is conversely consistent. Thus, $P^{\succsim}(c, E)=x=f(c, E)$.

\section{A.3 Proof of Theorem 2}

By Lemma 1, the PAP rules satisfy all of the axioms in Theorem 2. The proof that there are no other rules satisfying these axioms parallels that of Theorem 1 . However, the role of Lemma 4 in that proof is replaced by the following analogous result.

Lemma 5. Suppose that rule $f$ is continuous and satisfies any one of the following combinations of axioms:

(a) restricted additivity and restricted endowment additivity,

(b) restricted endowment additivity and dual restricted endowment additivity,

(c) restricted additivity and restricted multiplicative invariance.

Then, for each $\{i, j\} \subseteq \mathbb{N}, f$ coincides with $P, D^{i j}$, or $D^{j i}$ for claims problems in $\mathcal{C}^{\{i, j\}} \cdot 15$

Proof. Let $f$ denote a continuous rule, let $N \in \mathcal{N}$ consist of two claimants, let $(c, E) \in \mathcal{C}^{N}$ be such that $0<f(c, E)<c$, and define $C=\sum_{N} c_{i}$.

We only need to prove that, if $f$ satisfies the axioms in (a), (b), or (c), then $f(c, E)=P(c, E)$. This is analogous to proving Claim 1 in Lemma 4; the proof of Claims 2, 3, and 4 in Lemma 4 then goes through unchanged, thus proving Lemma 5 .

\footnotetext{
${ }^{15}$ Recall that $D^{i j}$ and $D^{j i}$ be as defined in (9).
} 
Suppose that $f$ satisfies the axioms in (a). For each $\lambda \in[0,1]$, let $E^{\lambda}=\lambda C+(1-\lambda) E$. Since the combination of endowment continuity and restricted endowment additivity implies endowment monotonicity (as noted in page 756 of Harless, 2017), Thus, for each $\lambda \in[0,1], f\left(c, E^{\lambda}\right) \geq f(c, E)>0$. Thus, by the first statement in Lemma 3 ,

$$
\text { for each } \lambda \in[0,1], \quad f\left(c, E^{\lambda}\right)=\lambda f(c, C)+(1-\lambda) f(c, E) \text {. }
$$

Thus, $f(c, E)<c$ and $\lambda<1$ imply that $f\left(c, E^{\lambda}\right)<c$. Thus, by Lemma 1 in Harless (2017), for each $\alpha \in[0,1]$ and each $\lambda<1, f\left(c, \alpha E^{\lambda}\right)=\alpha f\left(c, E^{\lambda}\right)$. By endowment continuity, as $\lambda \rightarrow 1, f(c, \alpha C)=\alpha f(c, C)=\alpha c$. Letting $\alpha=\frac{E}{C}, f(c, E)=P(c, E)$.

Suppose that $f$ satisfies the axioms in (b). Let $f^{d}$ denote the dual of $f$. By duality, $f^{d}(c, C-E)=c-f(c, E)<c$. By duality, $f^{d}$ satisfies restricted endowment additivity. By Lemma 1 in Harless (2017), for each $\alpha \in[0,1]$,

$$
f^{d}(c, \alpha(C-E))=\alpha f^{d}(c, C-E) .
$$

By duality, for each $\alpha \in[0,1], c-f(c, C-\alpha(C-E))=\alpha(c-f(c, E))$. Letting $E^{\alpha}=(1-\alpha) C+\alpha E$ and rearranging, for each $\alpha \in[0,1]$,

$$
f\left(c, E^{\alpha}\right)=(1-\alpha) c+\alpha f(c, E) .
$$

Since $f(c, E)<c$, for each $\alpha>0, f\left(c, E^{\alpha}\right)<c$. Let $\alpha>0$. By Lemma 1 in Harless (2017), for each $\beta \in[0,1], f\left(c, \beta E^{\alpha}\right)=\beta f\left(c, E^{\alpha}\right)$. By endowment continuity, as $\alpha \rightarrow$ 0 , for each $\beta \in[0,1], f(c, \beta C)=\beta f(c, C)=\beta c$. Letting $\beta=\frac{E}{C}, f(c, E)=P(c, E)$.

Suppose that $f$ satisfies the axioms in (c). For each $\alpha \in[0,1]$, let

$$
E^{\alpha}=\alpha C+(1-\alpha) E \text { and } F^{\alpha}=\alpha 2 C+(1-\alpha) E .
$$

By Lemma 2, for each $\alpha \in[0,1], f\left(c, E^{\alpha}\right) \geq f(c, E)>0$. By the first statement in Lemma 3 , for each $\alpha \in[0,1]$,

$$
f\left(c, E^{\alpha}\right)=\alpha f(c, C)+(1-\alpha) f(c, E)=\alpha c+(1-\alpha) f(c, E) .
$$

By restricted multiplicative invariance, $f(c, E)<c$ implies $f(2 c, E)=f(c, E)>0$. By Lemma 2, for each $\alpha \in[0,1], f\left(2 c, F^{\alpha}\right) \geq f(2 c, E)>0$. By the first statement in Lemma 3 , for each $\alpha \in[0,1]$,

$$
f\left(2 c, F^{\alpha}\right)=\alpha f(2 c, C)+(1-\alpha) f(2 c, E)=\alpha 2 c+(1-\alpha) f(2 c, E) .
$$

Let $\alpha \in(0,1)$. Let $\beta$ be such that $E^{\alpha}=F^{\beta}$; equivalently, $\beta=\frac{E^{\alpha}-E}{2 C-E}$. By (15), $f\left(c, E^{\alpha}\right)<c$. Thus, by restricted multiplicative invariance, $f\left(2 c, E^{\alpha}\right)=f\left(c, E^{\alpha}\right)$. Thus, $f\left(2 c, F^{\beta}\right)=f\left(c, E^{\alpha}\right)$. Thus, combining, (15) and (16), and noting that $f(2 c, E)=f(c, E), \beta 2 c+(1-\beta) f(c, E)=\alpha 2 c+(1-\alpha) f(c, E)$. Solving, $f(c, E)=$ $\frac{\alpha-2 \beta}{\alpha-\beta} c$. Plugging in $\beta=\frac{E^{\alpha}-E}{2 C-E}$ and $E^{\alpha}=\alpha C+(1-\alpha) E$ and simplifying, $f(c, E)=$ $\frac{E}{C} c=P(c, E)$. 


\section{A.4 Logical independence and robustness}

We introduce one further rule to illustrate the logical independence of the axioms in Theorems 1 and 2. Let $G$ denote the rule that coincides with the proportional rule for all claims problems with the exception of those involving only claimants $i, j \in \mathbb{N}$ where it maximizes $i$ 's award. Formally, for each $N \in \mathcal{N}$ such that $N \neq\{i, j\}$ and each $(c, E) \in \mathcal{C}^{N}, G(c, E)=P(c, E)$ and, for each $(c, E) \in \mathcal{C}^{\{i, j\}}, G(c, E)=D^{i j}(c, E)$ where $D^{i j}$ is as defined according to (9) in Subsection 3.1.

Table 2 below shows that non-PAP rules becomes admissible after dropping any one of the axioms in the characterizations given in Theorems 1 and $2 .{ }^{16}$

\begin{tabular}{l|cccc} 
& $C E L$ & $C E A$ & $L C F$ & $G$ \\
\hline bilateral consistency & $\checkmark$ & $\checkmark$ & $\checkmark$ & $\boldsymbol{x}$ \\
continuity & $\checkmark$ & $\checkmark$ & $\boldsymbol{x}$ & $\checkmark$ \\
restricted additivity & $\checkmark$ & $\boldsymbol{x}$ & $\checkmark$ & $\checkmark$ \\
dual restricted additivity & $\boldsymbol{x}$ & $\checkmark$ & $\checkmark$ & $\checkmark$ \\
restricted endowment additivity & $\boldsymbol{x}$ & $\checkmark$ & $\checkmark$ & $\checkmark$ \\
dual restricted endowment additivity & $\checkmark$ & $\boldsymbol{x}$ & $\checkmark$ & $\checkmark$ \\
restricted multiplicative invariance & $\boldsymbol{x}$ & $\checkmark$ & $\checkmark$ & $\checkmark$
\end{tabular}

Table 2

Finally, we show that weakening our additivity axioms in a natural way no longer enables us to characterize the PAP rules. Both restricted additivity and dual restricted additivity imply the following axiom: For each pair $c \in \mathbb{R}^{N}$ and each pair $E, E^{\prime} \in\left[0, \sum_{N} c_{i}\right]$ such that $0<f(c, E)<c$ and $0<f\left(c, E^{\prime}\right)<c$, $f(c, E)+f\left(c, E^{\prime}\right)=f\left(c+c, E+E^{\prime}\right)$. This axiom is satisfied by all the asymmetric rationing rules (Moulin, 2000), a class containing a number of continuous and consistent rules beyond the PAP rules.

\footnotetext{
${ }^{16}$ The priority-augmented weighted CEA rules and the priority-augmented weighted CEL rules characterized by Flores-Szwagrzak (2015) could also be used as examples in the table instead of the CEA and CEL rules, respectively. Rule $G$ could be replaced by any rule obtained by applying a different PAP rule to each $N \in \mathcal{N}$.
} 